\title{
Allan deviation tells the binding properties in single-molecule sensing with whispering-gallery-mode optical microcavities
}

\author{
Deshui Yu 10 and Frank Vollmer \\ Living Systems Institute, Physics and Astronomy, University of Exeter, Exeter EX4 4QD, United Kingdom
}

(Received 16 December 2020; revised 24 March 2021; accepted 29 March 2021; published 3 May 2021)

\begin{abstract}
We propose a scheme of exploring the kinetic properties of the receptor-ligand binding process by means of the frequency stability measurement of a whispering-gallery-mode optical microcavity. In this scheme, the probe beam is directly locked to the microcavity and the time resolution of the sensing detection is determined by the feedback loop response time $10 \mu \mathrm{s}$, which is much shorter than the current limit of about $20 \mathrm{~ms}$. The receptor-ligand interactions are entirely mapped onto the probe light frequency and can be recovered through the analysis of its Allan deviation. As a result, one can investigate a chemical and biological reaction at the single-molecule level without the need for resolving the occurrence and duration of individual binding events. We numerically study this sensing scheme based on a practical optoplasmonic platform, where several gold nanorods are adsorbed onto the surface of a spherical microcavity that is located in the aqueous environment. The microsphere has a typical radius of $40 \mu \mathrm{m}$ and quality factor of $10^{6}$ (finesse of $2.6 \times 10^{3}$ ) at the wavelength of $785 \mathrm{~nm}$. This powerful scheme may extend single-molecule sensing to the kinetic regime of the biochemical reactions.
\end{abstract}

DOI: 10.1103/PhysRevResearch.3.023087

\section{INTRODUCTION}

Owing to the properties of tight light confinement and high $Q$ factor, whispering-gallery-mode (WGM) microcavities have been extensively used in chemical and biological sensing [1]. The fundamental sensing mechanism lies in the spectral shift $\delta \lambda$ and broadening of a WGM induced by the variation in the refractive index of the surrounding environment of a passive/active microcavity [2,3]. For a WGM at the wavelength $\lambda_{\text {cav }}$, a dielectric nanoparticle with an excess polarizability $\alpha_{\mathrm{ex}}$ and a volume $V_{p}$ leads to

$$
\frac{\delta \lambda}{\lambda_{\text {cav }}}=\frac{V_{p}}{V_{\text {cav }}} \frac{\alpha_{\mathrm{ex}}}{2 \varepsilon_{0}} \text { with } V_{\text {cav }}=\frac{\int \epsilon(\mathbf{r})|\mathbf{E}(\mathbf{r})|^{2} d \mathbf{r}}{\left|\mathbf{E}\left(\mathbf{r}_{0}\right)\right|^{2}},
$$

where $\varepsilon_{0}$ is the vacuum permittivity, $\epsilon(\mathbf{r})$ denotes the spatial distribution of the relative permittivity, and $\mathbf{E}(\mathbf{r})$ represents the WGM field. The mode volume $V_{\text {cav }}$ of the WGM is evaluated based on the field intensity at the nanoparticle's position $\mathbf{r}_{0}$. Measuring $\delta \lambda$ allows one to estimate $\alpha_{\text {ex }}$ or the nanoparticle's size. For example, a silica (refractive index of 1.45) microsphere has a typical radius $R_{\mathrm{cav}}=40 \mu \mathrm{m}$ and is situated in the air. The mode volume of the fundamental TE-polarized WGM at $\lambda_{\text {cav }}=785 \mathrm{~nm}$ is computed as $V_{\text {cav }}=2 \times 10^{3} \mu \mathrm{m}^{3}$. A spherical polystyrene nanoparticle with a radius of $50 \mathrm{~nm}$ and $\alpha_{\mathrm{ex}} / \varepsilon_{0}=1.2$ yields $\delta \lambda=40 \mathrm{fm}$,

Published by the American Physical Society under the terms of the Creative Commons Attribution 4.0 International license. Further distribution of this work must maintain attribution to the author(s) and the published article's title, journal citation, and DOI. i.e., $\left|\delta \lambda / \lambda_{\text {cav }}\right| \sim 10^{-7}$. Besides dielectric nanoparticles, this mode-shift sensing mechanism is also applied to detect large single bioparticles, such as influenza viruses with $\left|\delta \lambda / \lambda_{\text {cav }}\right| \sim$ $10^{-8}$ [4]. However, detecting small single biomolecules (e.g., proteins and short DNA) demands a further suppression of $V_{\text {cav }}$.

Reducing $R_{\text {cav }}$ can strengthen the evanescent field $\mathbf{E}\left(\mathbf{r}_{0}\right)$, thereby suppressing $V_{\text {cav }}$ and enhancing $\delta \lambda[3,4]$. However, the microcavity's $Q$ factor also suffers from a large degradation due to increased radiation and surface scattering losses. An efficient measurement on $\delta \lambda$ requires the $Q$ factor (linewidth $\kappa_{0}=\omega_{\text {cav }} / Q$ with $\left.\omega_{\text {cav }}=2 \pi c / \lambda_{\text {cav }}\right)$ of a microcavity to be sufficiently high (narrow). Generally, the $Q$ factor of a WGM microcavity in the air reaches over $10^{8}$ [5-7], corresponding to a finesse $F=2.6 \times 10^{5}$ and linewidth $\kappa_{0}=2 \pi \times 4 \mathrm{MHz}$ of a WGM at $\lambda_{\text {cav }}=785 \mathrm{~nm}$ with $R_{\text {cav }}=40 \mu \mathrm{m}$. However, detecting single biomolecules usually requires the microcavity to be immersed in an aqueous solution. This results in a serious degradation of the microcavity's $Q$ factor, for instance, from $10^{8}$ in the air to $10^{6}$ in the water [8], because of the huge absorption losses in the aqueous environment. Reducing $R_{\text {cav }}$ may further degrade $Q$, thereby deteriorating the sensor's performance.

The mode volume $V_{\text {cav }}$ can, alternatively, be suppressed by hybridizing the localized surface plasmon resonance (LSPR), i.e., the collective oscillation of free electrons in nanostructured metals, with WGM microcavities [Fig. 1(a)]. The LSPR allows the subwavelength light confinement beyond the diffraction limit, boosting the local evanescent field $\mathbf{E}\left(\mathbf{r}_{0}\right)$. For example, a gold nanorod, whose circular cross-section diameter and length are $10 \mathrm{~nm}$ and $35 \mathrm{~nm}$, respectively, gives a near-field intensity enhancement factor $\Lambda \sim 10^{3}$ around two hotspots [9]. Here, the plasmonic hotspots denote the regions 

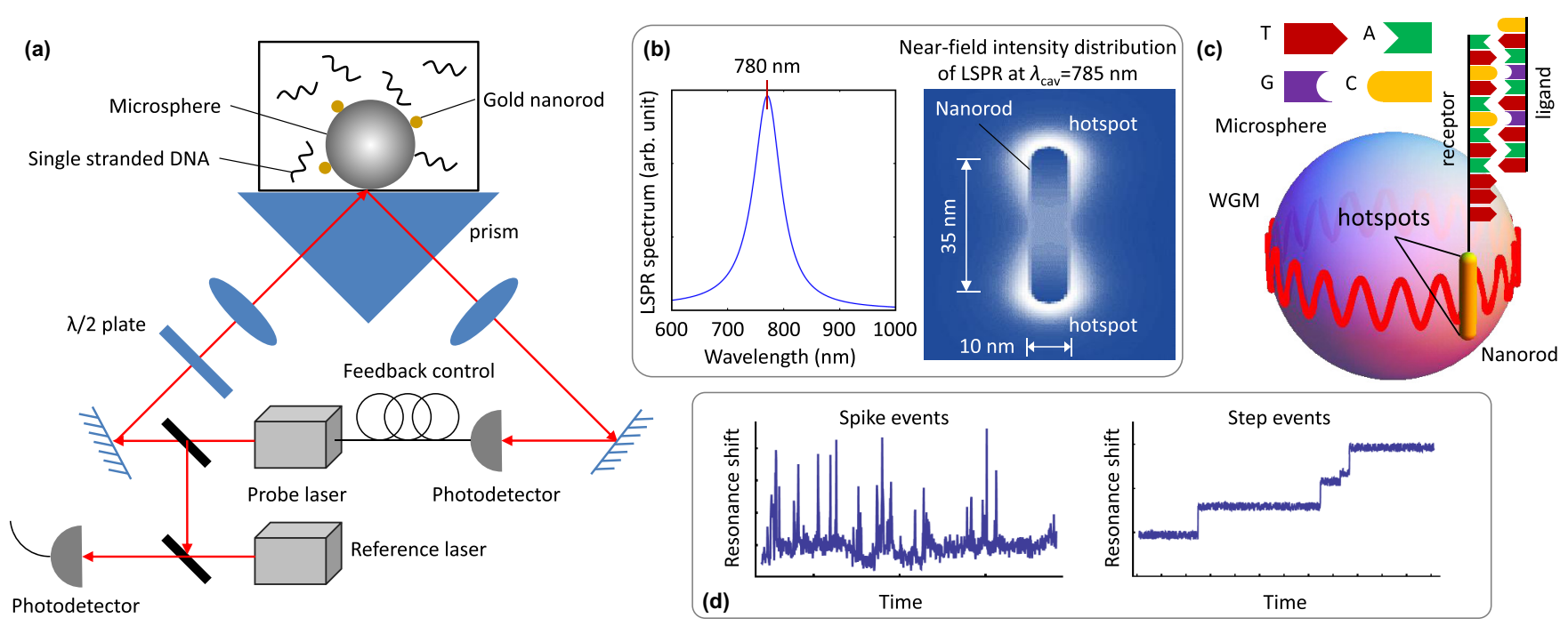

FIG. 1. (a) Single-molecule detection based on the optoplasmonic hybridization. A silica microsphere with a radius of $R_{\text {cav }}=40 \mu \mathrm{m}$ is situated in the aqueous environment. Several gold nanorods are adsorbed onto the microsphere's surface. The TE-polarized cw and ccw WGMs at $\lambda_{\text {cav }}=785 \mathrm{~nm}$ are excited by a probe laser through an optical prism. The transmitted light is fed back into the probe laser, locking its frequency to the microcavity. The Allan deviation of the probe laser is measured by the beat note with a reference laser. (b) Longitudinal LSPR spectrum of a gold nanorod and the electric-field intensity distribution of LSPR at $\lambda_{\text {cav }}$. The circular cross section of the nanorod has a diameter of $10 \mathrm{~nm}$ and the nanorod's length is $35 \mathrm{~nm}$. The regions around two ends of the nanorod are termed the hotspots, where the near-field intensity is strongly enhanced by a factor $\Lambda=800$. (c) Evanescent interaction between the microsphere and a gold nanorod. The receptor molecules, which interact with the ligand molecules, are permanently attached within the nanorod's hotspots. (d) Schematic diagrams of the time traces of the wavelength shift of a WGM. The spike events correspond to the transient receptor-ligand interactions while the step events denote the permanent binding between single receptor and ligand molecules.

near sharp corners and tips of metal nanoparticles [Fig. 1(b)]. In addition, Ohmic losses of metal nanoparticles introduce an extra decay channel of intracavity photons, degrading the $Q$ factor of microcavities. For instance, binding five gold nanorods, which are all aligned perpendicularly to the equatorial WGM, onto a microsphere immersed in the water may reduce $Q$ from $1 \times 10^{6}$ to $5 \times 10^{5}$. Nevertheless, the large intensity enhancement factor $\Lambda$ still strongly enhances the sensor's sensitivity.

Moreover, modifying the surface of metal nanoparticles with receptor $(\mathrm{R})$ molecules enables a label-free detection of specific ligand (L) molecules [Fig. 1(c)] through the relevant binding reaction,

$$
\mathrm{R}+\mathrm{L} \underset{k_{\text {off }}}{\stackrel{k_{\mathrm{on}}}{\rightleftharpoons}} \mathrm{RL},
$$

much simplifying the sensing procedures. Here, $k_{\text {on }}$ (in units of $\mathrm{M}^{-1} \mathrm{~s}^{-1}$, where $\mathrm{M}$ denotes molar) and $k_{\text {off }}$ (in units of $\mathrm{s}^{-1}$ ) are the on-rate and off-rate constants, respectively. The singlemolecule detection based on the optoplasmonic hybridization has been demonstrated for short DNA oligonucleotides [9], bovine serum albumin protein molecules [10], and even atomic ions, such as $\mathrm{Zn}^{2+}$ and $\mathrm{Hg}^{2+}$ [11], whose ionic radii are 0.74 and $1.02 \AA$, respectively. This is the highest sensitivity achieved by WGM microcavities so far. Recently, these optoplasmonic devices have been widely applied to monitor the reaction kinetics between receptor and ligand molecules in a label-free manner [12-14].

For conventional optoplasmonic sensors, the transmission spectrum of a WGM microcavity is measured by sweeping the wavelength of a probe laser. The single-molecule interactions are identified by the spike and step events [Fig. 1(d)] extracted, i.e., through fitting the spectrum to a Lorentzian profile, from a time trace of the WGM wavelength $\lambda_{\text {cav }}$. When a ligand molecule binds to a receptor molecule, $\lambda_{\text {cav }}$ experiences a shift $\delta \lambda$ that is determined by Eq. (1). For a transient receptor-ligand interaction, $\delta \lambda$ vanishes once the ligand molecule is removed from the receptor molecule, leaving a spike in the time trace of $\lambda_{\text {cav }}$. By contrast, a step event occurs for a permanent receptor-ligand binding. The typical time resolution of the sensing detection is about tens of $\mathrm{ms}$ (e.g., $20 \mathrm{~ms}$ in [11-14]), which is limited by the scanning rate of the probe laser. To accurately capture the transmission spectrum of the microcavity, the probe laser frequency goes over the central frequency of the WGM with a width larger than $\kappa_{0}$. Also, the laser scanning should be slow enough that the intracavity field continuously maintains a steady state, i.e., adiabatic limit.

The adjacent single-molecule events with an interval shorter than the time resolution cannot be distinguished. Thus, the sensor's applications are restricted to an aqueous solution of ligand molecules with an appropriate off-rate constant $k_{\text {off }}$ (e.g., $k_{\text {off }}=47$ and $23 \mathrm{~s}^{-1}$ in [13]) and also at a suitably low concentration ( $\mathrm{fM} \sim \mathrm{nM}$ in [11-14]) with an appropriate onrate constant $k_{\text {on }}$. This weakness may be overcome by directly locking the probe beam to the microcavity. The frequency locking time can be as short as $10 \mu \mathrm{s}$ [15], leading to a threeorders-of-magnitude improvement in the time resolution.

Moreover, extracting the spike and step events from a time trace of the WGM wavelength is usually implemented in a manual manner [11-14]. An empirical algorithm is applied to remove the background fluctuations and drift of the probe 
laser [11]. However, it inevitably omits the weak spike/step events that are buried in the background noise and is thereby unable to reveal the entire picture of the chemical and biological reaction. To address this issue, the spike and step events may be treated as the extra noise to the microcavity and one performs the stability analysis on the probe beam whose frequency is locked to the microcavity.

In this paper, we numerically study a fast and sensitive single-molecule detection scheme. The probe beam is directly locked to the microcavity and the kinetic properties of the receptor-ligand binding interactions are explored by measuring the frequency stability, i.e., Allan deviation, of the probe light. This scheme is capable of capturing those single-molecule events with a duration of $10 \mu \mathrm{s}$, limited by the integration time of the feedback loop. The sensing method is applicable to the previously inaccessible kinetic regime with large on-rate $k_{\text {on }}$ (at a high ligand concentration) and off-rate $k_{\text {off }}$ constants.

\section{PHYSICAL MODEL}

The single-molecule sensing scheme is illustrated in Fig. 1(a). The light from a probe laser (amplitude $\mathcal{A}_{p}$ and frequency $\omega_{p}$ ) is evanescently coupled into a silica microsphere via the total internal reflection at the surface of an optical prism. The microsphere-prism coupling rate is $\kappa_{\text {in }}$. The microsphere with a typical radius $R_{\text {cav }}=40 \mu \mathrm{m}$ is immersed in the water (refractive index of $n=1.33$ ). The probe beam excites two degenerate fundamental clockwise (cw) and counterclockwise (ccw) WGMs (mode index $l=453$ ) at $\lambda_{\text {cav }}=$ $785 \mathrm{~nm}$. Both WGMs are TE-polarized and their quality factor in the aqueous environment approximates $Q=10^{6}$. The loss rate of two WGMs is $\kappa_{0}=\omega_{\text {cav }} / Q$ with $\omega_{\text {cav }}=2 \pi c / \lambda_{\text {cav }}$. We assume a weak microsphere-prism coupling, i.e., $\kappa_{\text {in }} \ll \kappa_{0}$. The mode volume of the microcavity is computed as $V_{\text {cav }}=$ $7.2 \times 10^{3} \mu \mathrm{m}^{3}$.

The light transmitted from the microsphere is measured by a photodetector. Unlike conventional optoplasmonic sensors [11-14], the output from the photodetector is further fed back into the probe laser system through a servo, locking the probe light frequency $\omega_{p}$ to the central frequency $\omega_{\text {cav }}$ of the ccw WGM. The characteristic time of the feedback loop can be as short as $10 \mu \mathrm{s}$ [15]. The frequency stability (i.e., Allan deviation) of the probe laser is assessed by the beat note between the locked probe laser and a high- $Q$ reference laser whose stability much exceeds both probe laser and microsphere [Fig. 1(a)]. Thus, the stability of the microcavity is indirectly measured.

The LSPR of gold nanorods (circular cross-section diameter of $10 \mathrm{~nm}$ and length of $35 \mathrm{~nm}$ ) is utilized to enhance the ligand molecule-microcavity interaction (i.e., optoplasmonic sensing). These metal nanoparticles are permanently adsorbed to the microsphere's surface [9] and coupled to the evanescent field of the microcavity, thereby boosting the near-field intensity within the hotspot regions [Fig. 1(b)]. Each nanorod has two hotspots. The surface of each nanorod is modified with many receptor molecules, enabling a label-free detection of specific ligand molecules [Fig. 1(c)]. In the following, we assume that the receptor-ligand pair consists of partially mismatched single DNA strands [9]. Nevertheless, the sensing scheme is applicable to other receptor-ligand pairs. We compute the longitudinal LSPR spectrum of the nanorod by using the finite-difference time-domain method. Figure 1(b) illustrates that the LSPR spectrum is peaked at $780 \mathrm{~nm}$. The nanorod's electric polarizability at $\lambda_{\text {cav }}$ is evaluated as $\alpha_{\mathrm{NR}}=0.96 \times 10^{-22}+i 1.73 \times 10^{-22} \mathrm{~m}^{3}$. The enhancement factor of the electric-field intensity around the nanorod's hotspots reaches $\Lambda=800$ [9].

The typical number of the adsorbed nanorods in experiment is $N=5$. In the ideal situation, the longitudinal direction of all nanorods is perpendicular to the equatorial plane of the microsphere. The complementary single DNA strands, i.e., ligands, are injected into the solution. Since the receptor and ligand strands are partially mismatched, the transient hybridization events [i.e., the spike events in the time trace of the WGM frequency as shown in Fig. 1(c)] occur primarily. We assume the sample solution is in the equilibrium state and neglect the permanent binding events [i.e., the step events as shown in Fig. 1(c)] in the following discussion. One ligand binding to one receptor located at a nanorod's hotspot gives rise to a polarizability increment, for instance, $\alpha_{\text {DNA }} \approx 2.5 \times 10^{-25} \mathrm{~m}^{3}$ for the short oligonucleotides [9], to the corresponding nanorod. Normally, such a small polarizability increment cannot be detected by the WGM microcavity in the absence of the LSPR.

The experimental measurement has demonstrated that two degenerate cw and ccw WGMs are indirectly coupled via the backscattering and Rayleigh scattering of the nanoparticles [16-18], leading to the mode shift and broadening and the degeneracy lifting. This can be described by the following equation of motion for the amplitudes $\mathcal{A}_{\mathrm{cw}, \mathrm{ccw}}$ of two WGMs (Appendix)

$$
\begin{aligned}
\left(\begin{array}{c}
\dot{\mathcal{A}}_{\mathrm{cw}} \\
\dot{\mathcal{A}}_{\mathrm{ccw}}
\end{array}\right)= & {\left[-\frac{\kappa}{2}+i\left(\omega_{p}-\omega_{\mathrm{cav}}\right)\right]\left(\begin{array}{c}
\mathcal{A}_{\mathrm{cw}} \\
\mathcal{A}_{\mathrm{ccw}}
\end{array}\right) } \\
& -\sum_{j=1}^{N}\left(\frac{\Gamma_{j}}{2}-i \Delta_{j}\right)\left(\begin{array}{cc}
1 & e^{i \phi_{j}} \\
e^{-i \phi_{j}} & 1
\end{array}\right)\left(\begin{array}{c}
\mathcal{A}_{\mathrm{cw}} \\
\mathcal{A}_{\mathrm{ccw}}
\end{array}\right) \\
& -\sqrt{\kappa_{\mathrm{in}}}\left(\begin{array}{c}
0 \\
\mathcal{A}_{p}
\end{array}\right),
\end{aligned}
$$

with $\kappa=\kappa_{0}+\kappa_{\text {in }}$. Here, $\phi_{j}$ is the phase difference between $\mathrm{cw}$ and ccw optical waves at the position of the $j$ th nanorod. In the numerical simulation, $\phi_{j}$ is produced according to the uniform distribution of the gold nanorods in the equatorial circumference of the microsphere. The probe beam $\mathcal{A}_{p}$ is only coupled with the ccw WGM. The Rayleigh scattering rate and mode frequency shift caused by the $j$ th nanorod are expressed as

$$
\Gamma_{j}=\frac{\left(\alpha_{\mathrm{NR}}^{2}+\Lambda \alpha_{j}^{2}\right) \omega_{\mathrm{cav}}^{4}}{6 \pi(c / n)^{3} V_{\mathrm{cav}}}, \quad \Delta_{j}=\frac{\left(\alpha_{\mathrm{NR}}+\Lambda \alpha_{j}\right) \omega_{\mathrm{cav}}}{2 V_{\mathrm{cav}}},
$$

respectively. The extra time-dependent polarizability of the $j$ th nanorod is given by

$$
\alpha_{j}=\sum_{k}\left[\Theta\left(t-t_{j, k}^{\mathrm{on}}\right)-\Theta\left(t-t_{j, k}^{\mathrm{off}}\right)\right] \alpha_{\mathrm{DNA}},
$$

where $\Theta(t)$ is the unit step function and $t_{j, k}^{\text {on }}\left(t_{j, k}^{\text {off }}\right)$ denotes the initial (end) time of the binding interaction between the $k$ th ligand and a receptor on the $j$ th nanorod. 
(a)

- Bare microsphere in the water

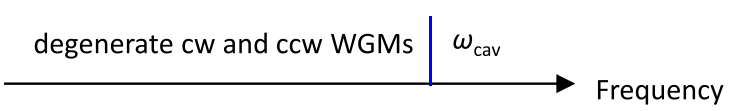

- Microsphere + gold nanorods + ligand molecules
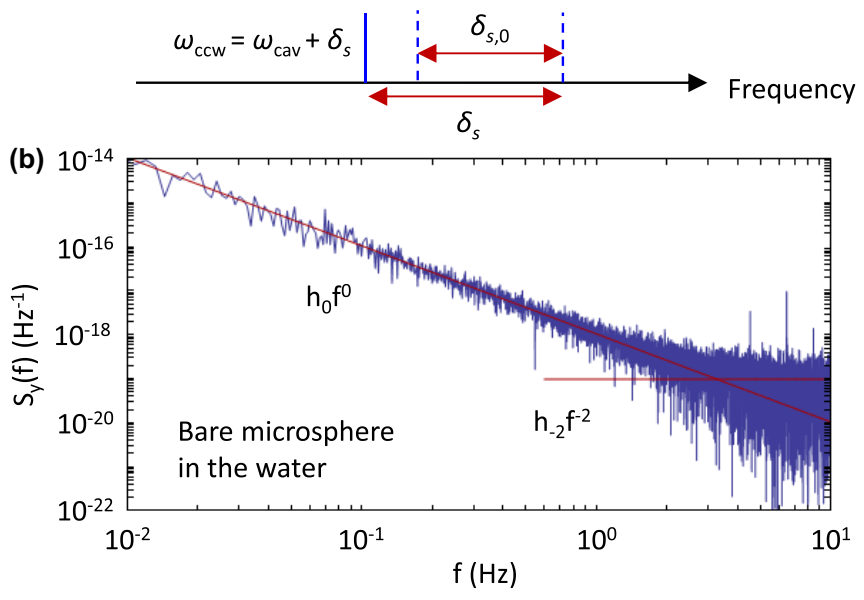

(c)

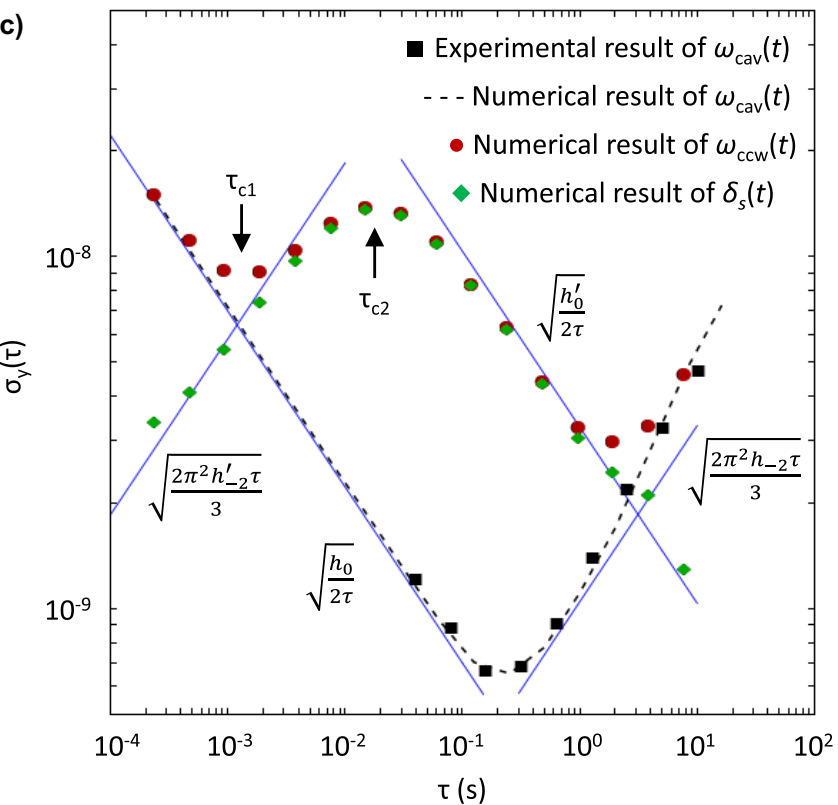

FIG. 2. (a) Frequency relations. The $\mathrm{cw}$ and $\mathrm{ccw}$ WGMs are degenerate for the bare microsphere in the water. The resonance frequency of two WGMs is $\omega_{\text {cav }}$. When the gold nanorods are adsorbed to the surface of the microsphere, the resonance frequency $\omega_{\text {ccw }}$ of the ccw WGM is shifted from $\omega_{\mathrm{cav}}$ by an amount of $\delta_{s, 0}$. When the ligand molecules further bind to the gold nanorods, the total frequency shift of the ccw WGM is $\delta_{s}$, i.e., $\omega_{\text {ccw }}=\omega_{\text {cav }}+\delta_{s}$. (b) Typical power spectral density $S_{y}(f)$ of the frequency noise in $\omega_{\text {cav }}(t)$ measured in experiment. The bare microsphere, i.e., without the gold nanorods and in the absence of the receptor-ligand binding interactions, is immersed in the water. (c) Allan deviation $\sigma_{y}(\tau)$. The square symbol denotes the stability of the experimentally-measured frequency $\omega_{\text {cav }}$ of the microsphere in the aqueous environment. The corresponding numerical simulation result is given by the dashed line. The circle symbol is the numerical result of the spike-event-perturbed frequency $\omega_{\mathrm{ccw}}$ with $R=250 \mathrm{~s}^{-1}, T=244 \mathrm{~s}$, and $\tau_{\text {ave }}=10^{-2} \mathrm{~s}$. The diamond symbol corresponds to the numerical simulation result of the spike-event-induced frequency shift $\delta_{s}$. The solid curves denote the asymptotes.

The coupling between cw and ccw WGMs lifts their degeneracy. We focus on the ccw WGM since the probe laser is locked to it. The transmission of the ccw wave

$$
S_{\text {Tran }}\left(\omega_{p}\right)=\frac{\left|\mathcal{A}_{\mathrm{ccw}}(t)\right|^{2}}{\kappa_{\text {in }}\left|\mathcal{A}_{p}\right|^{2}},
$$

as a function of the probe light frequency $\omega_{p}$ can be derived from Eq. (3). The spectrum $S_{\text {Tran }}\left(\omega_{p}\right)$ is single-peaked at $\left(\omega_{\text {cav }}+\delta_{s}\right)$ with a linewidth $\left(\kappa+\delta_{b}\right)$. That is to say, the mode splitting is unresolvable due to the relatively large cavity loss rate $\kappa$ [19]. The extra spectral shift $\delta_{s}(t)$ and broadening $\delta_{b}(t)$ are caused by the nanorods and the receptor-ligand binding interactions. In what follows, we may set $\omega_{p}=\omega_{\mathrm{ccw}}$ with the central frequency of the ccw WGM

$$
\omega_{\mathrm{ccw}}(t)=\omega_{\mathrm{cav}}(t)+\delta_{s}(t),
$$

for the reasons: (1) The frequency locking time of the feedback loop is much shorter than the time scale of interest; and (2) The extra servo and photon shot noises are sufficiently low. Figure 2(a) summaries the relations among $\omega_{\mathrm{cav}}, \omega_{\mathrm{ccw}}$, and $\delta_{s}$. We use $\delta_{s, 0}$ to refer to the mode-shift component caused solely by the gold nanorods in the absence of the receptor-ligand interactions.

Next, we consider the numerical simulation of the receptorligand binding events. The experimental observations [11-14] have manifested: (1) The statistics of the transient singlemolecule events follow the Poisson distribution, that is, the probability of $K \in \mathbb{Z}$ spike events occurring within a fixed period $T$ is given by

$$
p(K)=\frac{(R T)^{K} e^{-R T}}{K !} .
$$

The total event rate is equal to $R=\sum_{j=1}^{N} r_{j}$ (in units of $\mathrm{s}^{-1}$ ) with the event rate $r_{j}$ of the $j$ th nanorod. We neglect the difference between gold nanorods and have $R=N r$ with $r=$ $r_{j}$. The typical $R$ in the conventional optoplasmonic sensing experiments is less than $1 \mathrm{~s}^{-1}$ for various ligand molecules $[11,12]$. Raising the ligand concentration enhances $R$. However, the higher $R$ regime has thus far been difficult to analyze for the reason of the limited time resolution. Distinguishing the single-molecule events requires the time resolution to be much shorter than $R^{-1}$. The time resolution of the conventional optoplasmonic sensors is limited by $\sim 20 \mathrm{~ms}$ because of the slow scanning rate of the probe beam [11-14]; and (2) The duration $\left(t_{j, k}^{\text {off }}-t_{j, k}^{\text {on }}\right)$ of the single-molecule binding interaction follows the exponential distribution with an average duration $\tau_{\text {ave }}$. When $\tau_{\text {ave }}<R^{-1}$, different single-molecule events are distinguishable. By contrast, different single-molecule events are overlapped when $\tau_{\text {ave }}>R^{-1}$. Again, the current experimental detection is limited by $\tau_{\text {ave }} \sim 20 \mathrm{~ms}$. As we will see below, our sensing scheme may overcome this restriction.

Indeed, $R$ and $\tau_{\text {ave }}$ are directly linked to the kinetic on-rate $k_{\text {on }}=R / c[11]$ and off-rate $k_{\text {off }}=1 / \tau_{\text {ave }}$ [13] constants. Here, $c$ denotes the ligand concentration. In some literature, both $k_{\text {on }}$ and $k_{\text {off }}$ are normalized per nanorod. Measuring $R$ and $\tau_{\text {ave }}$ allows one to derive the kinetic properties of the receptor-ligand 
binding interactions. For examples, conventional optoplasmonic sensing experiments have measured $k_{\text {on }}=0.53 \times 10^{3}$ and $3.8 \times 10^{3} \mathrm{M}^{-1} \mathrm{~s}^{-1}$ for the gold nanorods interacting with $\mathrm{Hg}^{2+}$ and $\mathrm{Zn}^{2+}$ ions [11], respectively, and $k_{\text {off }}=47$ and $23 \mathrm{~s}^{-1}$ for the primer/template DNA interacting with Thermus aquaticus and Klenow fragment DNA polymerases [13], respectively.

The above two experimental manifestations allow one to numerically predict $t_{j, k}^{\text {on }}$ and $t_{j, k}^{\text {off }}$ in a time trace (length of $T$ ) of $\omega_{\mathrm{ccw}}(t)$. The specific numerical simulation processes are summarized as follows: (1) Generate a series of random times (number of $K$ ) according to the Poisson distribution with the event rate $R$ and the time length $T$. Each time point corresponds to the initial time of a spike event; (2) Create $K$ uniformly distributed random integers within $[1, N]$ and map this array onto the time series generated in the step (1). Thus, one obtains the initial times of different single-molecule binding events in Eq. (5), $t_{j, k}^{\text {on }}$ with $j \in[1, N]$ and $k \in[1, K]$; and (3) Generate a series of random time intervals $\tau_{k}$ according to the exponential distribution with the average duration $\tau_{\text {ave }}$. As a result, the end times of different binding events in Eq. (5) are given by $t_{j, k}^{\text {off }}=t_{j, k}^{\text {on }}+\tau_{k}$. Once obtaining $t_{j, k}^{\text {on }}$ and $t_{j, k}^{\text {off }}$, one may simulate the time-dependent central frequency $\omega_{\mathrm{ccw}}(t)$ of the ccw WGM through Eqs. (3)-(5).

\section{RESULTS AND ANALYSIS}

Let us focus on the stability of the frequency fluctuation signal

$$
y(t)=\omega_{\mathrm{ccw}}(t) / \omega_{0}-1,
$$

whose power spectral density is given by

$$
S_{y}(f)=|\mathcal{F}[y(t)]|^{2}=\left|\int y(t) e^{-i 2 \pi f t} d t\right|^{2} .
$$

Here, $\omega_{0}=2 \pi \times 382 \mathrm{THz}$ is the constant central frequency of the ccw WGM. We first consider $y(t)$ in the absence of $\delta_{s}(t)$ and the reduced signal $y(t)=\omega_{\text {cav }}(t) / \omega_{0}-1$ with $\delta_{s}=$ 0 represents a bare microsphere in the aqueous environment. Figure 2(b) shows a typical $S_{y}(f)$ of the bare microsphere measured in experiment. It is seen that $y(t)$ is mainly composed of the white frequency $\left(\propto f^{0}\right)$ and random walk $(\propto$ $f^{-2}$ ) noises. The stability of $y(t)$ is evaluated by the Allan deviation $\sigma_{y}(\tau)$ with a sampling time $\tau$ [20,21]. The white frequency noise originates from the microsphere's thermal Brownian motion $[22,23]$ that leads to a $\frac{1}{\sqrt{\tau}}$-dependence of the short-term $(\tau<0.1 \mathrm{~s})$ stability [24],

$$
\sigma_{y}(\tau)=\sqrt{\frac{h_{0}}{2 \tau}} .
$$

By contrast, the random walk noise arises from the linear frequency drift and raises $\sigma_{y}(\tau>0.1 \mathrm{~s})$ with a scaling factor $\sqrt{\tau}[24]$

$$
\sigma_{y}(\tau)=\sqrt{\frac{2 \pi^{2} h_{-2} \tau}{3}} .
$$

Additionally, $\sigma_{y}(\tau)$ displayed in Fig. 2(c) manifests the existence of the flicker walk $\left(\propto f^{-3}\right)$ component, which gives $\sigma_{y}(\tau) \propto \tau$ for a large $\tau$, in $y(t)$. As a result, $S_{y}(f)$ with $\delta_{s}(t)=0$ (i.e., the bare microsphere in the aqueous environment) takes the form

$$
S_{y}(f)=h_{0} f^{0}+h_{-2} f^{-2}+h_{-3} f^{-3},
$$

with the typical values $h_{0}=0.98 \times 10^{-19} \mathrm{~Hz}^{-1}, h_{-2}=$ $1.67 \times 10^{-19} \mathrm{~Hz}$, and $h_{-3}=2.22 \times 10^{-19} \mathrm{~Hz}^{2}$ derived from the curve fitting. We have also estimated the fundamental thermorefractive-noise-limited stability [25,26],

$$
\sigma_{y}^{\mathrm{TR}}(\tau)=\frac{4 \times 10^{-13}}{\sqrt{\tau}},
$$

which is much lower than $\sqrt{h_{0} / 2 \tau}$. According to $S_{y}(f)$ with $\delta_{s}(t)=0$, one may numerically generate the noise-perturbed $\omega_{\text {cav }}(t)$ via the method of digitally filtering the white noise [27]. As shown in Fig. 2(c), the Allan deviation of the numerically generated $\omega_{\text {cav }}(t)$ well matches the experimental measurement.

After obtaining $t_{j, k}^{\text {on }}, t_{j, k}^{\text {off }}$, and $\omega_{\text {cav }}(t)$, we numerically simulate the time trace of the mode frequency $\omega_{\mathrm{ccw}}(t)$ by means of calculating the transmission spectrum $S_{\text {Tran }}\left(\omega_{p}=\omega_{\text {ccw }}\right)$ from Eqs. (3)-(6). In the presence of the transient spike events [i.e., $\delta_{s}(t) \neq 0$ ], the Allan deviation of $y(t)$ exhibits a distinct behavior compared to that of $\delta_{s}(t)=0$. As shown in Fig. 2(c), $\sigma_{y}(\tau)$ for $\omega_{\mathrm{ccw}}(t)$ with $\delta_{s}(t) \neq 0$ goes up firstly at the sampling time $\tau=\tau_{c 1}$ and then degrades at $\tau=\tau_{c 2}$. Understanding the underlying mechanism relies on extracting the spike-event noise $\delta_{s}(t)$ from $\omega_{\mathrm{ccw}}(t)$. This is feasible in the numerical simulation but challenging in experiment.

As depicted in Fig. 3(a), multiple spikes are presented in the time trace of $\delta_{s}(t)$, where we have removed the constant bias $\delta_{s, 0}$ caused by the gold nanorods. The Fourier analysis

$$
S_{\left(\delta_{s} / \omega_{0}\right)}(f)=\left|\mathcal{F}\left[\delta_{s}(t) / \omega_{0}\right]\right|^{2},
$$

illustrates that $\delta_{s}(t)$ predominantly consists of the white frequency $h_{0}^{\prime} f^{0}$ and random walk $h_{-2}^{\prime} f^{-2}$ noises [Fig. 3(b)],

$$
S_{\left(\delta_{s} / \omega_{0}\right)}(f)=h_{0}^{\prime} f^{0}+h_{-2}^{\prime} f^{-2} .
$$

The cross between $h_{0}^{\prime} f^{0}$ and $h_{-2}^{\prime} f^{-2}$ occurs at

$$
f_{c 2}=\frac{\sqrt{3}}{2 \pi \tau_{c 2}} .
$$

The random walk component strongly raises the Allan deviation of $\delta_{s}(t) / \omega_{0}$, i.e., following the $\sqrt{2 \pi^{2} h_{-2}^{\prime} \tau / 3}$ line, until hitting the $\sqrt{h_{0}^{\prime} / 2 \tau}$ line that is determined by the white frequency noise [Fig. 2(c)]. This coincides with the corresponding behavior of the Allan deviation $\sigma_{y}(\tau)$ of $\omega_{\mathrm{ccw}}(t)$ with $\delta_{s}(t) \neq 0$.

Using Eq. (3), one predicts that a ligand interacting with a receptor gives rise to a $10-\mathrm{fm}$ increment of $\delta_{s}$. That is, the single-molecule-induced mode shift is $10 \mathrm{fm}$. The spike heights in Fig. 3(a) are closely related to the single-molecule event rate $R$ and the average event duration $\tau_{\text {ave. The re- }}$ ciprocal of $R$ measures the average interval between the initial times of two adjacent receptor-ligand binding events. When $R^{-1}$ exceeds the average duration $\tau_{\text {ave }}$, a ligand may start to interact with a receptor before the end of another receptor-ligand binding event. This results in an overlap of different binding events, i.e., multimolecular events, and a 

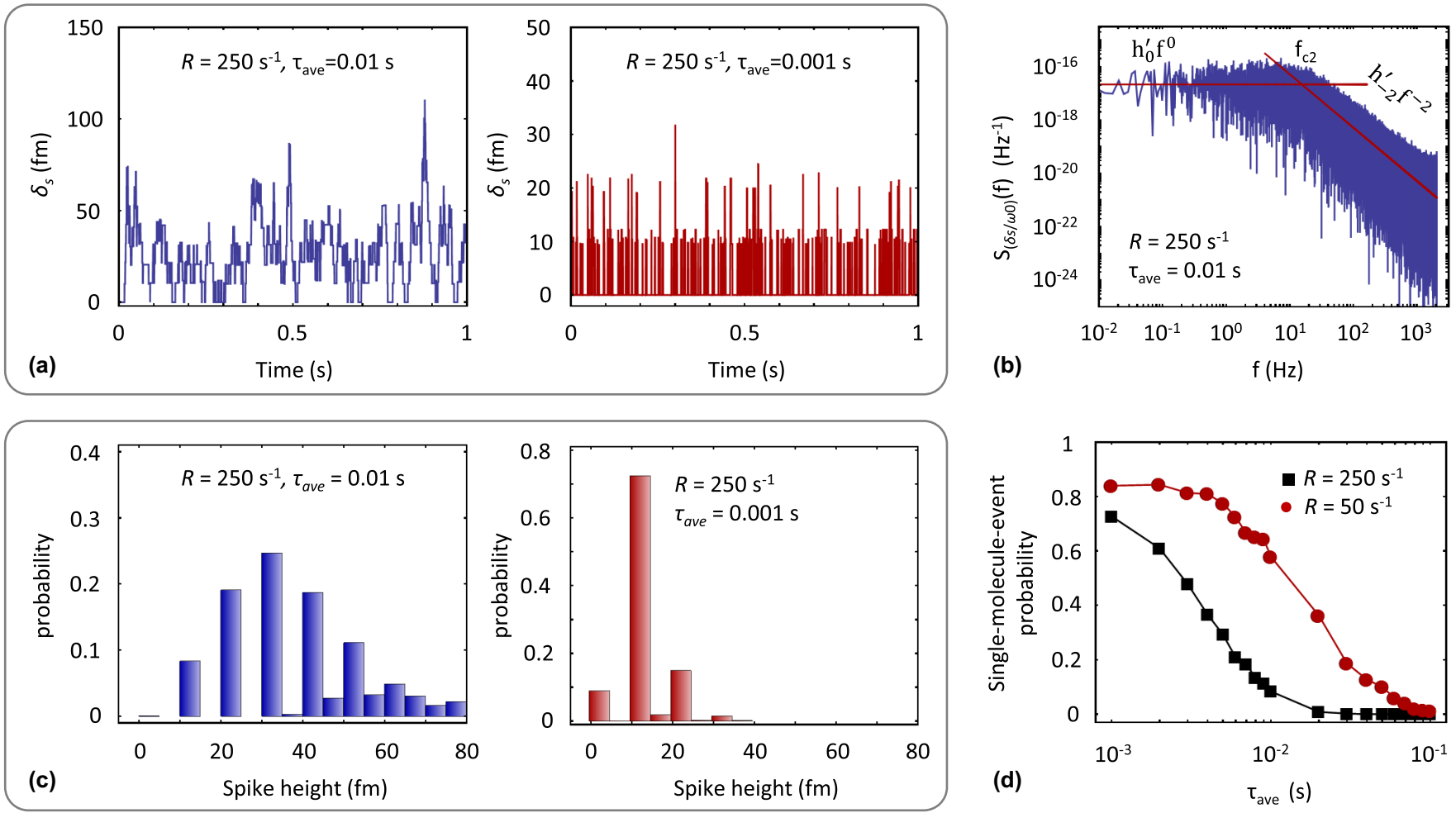

FIG. 3. (a) Numerically simulated traces of the spike-event-induced mode shift $\delta_{s}(t)$ in units of fm. The constant bias $\delta_{s, 0}$ caused by the gold nanorods has been removed. A ligand binding to a receptor causes an about 10-fm shift. (b) Power spectral density $S_{\left(\delta_{s} / \omega_{0}\right)}(f)$ of the numerically simulated $\delta_{s}(t)$. The curve fitting illustrates $S_{\left(\delta_{s} / \omega_{0}\right)}(f)=h_{0}^{\prime} f^{0}+h_{-2}^{\prime} f^{-2}$ with $h_{0}^{\prime}=2.14 \times 10^{-17} \mathrm{~Hz}^{-1}$ and $h_{-2}^{\prime}=5.20 \times$ $10^{-15} \mathrm{~Hz}$. (c) Distribution of spike heights extracted from the numerical simulation. The bins around $10 \mathrm{fm}$ correspond to the single-molecule events. (d) Probability of the single-molecule events extracted from the numerical simulation vs the average duration $\tau_{\text {ave }}$ for different event rates $R$.

spike higher than $10 \mathrm{fm}$. Figure 3(c) displays the statistical distribution of the spike heights extracted from Fig. 3(a). It is seen that when $R \tau_{\text {ave }}>1$ the distribution is maximized at a spike height of about $R \tau_{\text {ave }} \times 10 \mathrm{fm}$. By contrast, the distribution is maximized at $10 \mathrm{fm}$ for $R \tau_{\text {ave }}<1$, indicating that the single-molecule events take the predominant role.

Figure 3(d) illustrates the dependence of the singlemolecule-event probability on the average duration $\tau_{\text {ave }}$. A short $\tau_{\text {ave }}$ highlights the probability of the single-molecule events. Shortening $\tau_{\text {ave }}$ relies on weakening the receptorligand interaction, for instance, increasing the number of mismatched bases between receptor and ligand single DNA strands [9]. However, a short $\tau_{\text {ave }}$ also demands a fast feedback loop. The frequency locking time of $10 \mu \mathrm{s}$ is feasible in experiment [15]. Thus, $\tau_{\text {ave }}$ can be, in principle, as short as $10 \mu \mathrm{s}$, which is over $10^{3}$ times smaller than the current resolution time limit $\sim 20 \mathrm{~ms}$ of the conventional optoplasmonic sensors [11-14].

We use $\Delta_{\text {ave }}$ to denote the average height of different spikes in a time trace of $\delta_{s}(t)$. It is impractical to directly derive the analytical expression of $\Delta_{\text {ave }}$. Here, we introduce the empirical equation

$$
\Delta_{\mathrm{ave}}=\left[\left(R \tau_{\mathrm{ave}}\right)+\left(1+R \tau_{\mathrm{ave}}\right)^{-1 / 2 m}\right] \times 10 \mathrm{fm},
$$

where the curve fitting gives $m=2$. The validity of this expression is confirmed by Fig. 4(a). In the limit of $R \tau_{\text {ave }} \ll 1$, Eq. (18) is reduced to $\Delta_{\text {ave }} \approx 10 \mathrm{fm}$, i.e., the single-molecule spike events predominate. By contrast, $R \tau_{\text {ave }} \gg 1$ leads to $\Delta_{\text {ave }} \approx\left(R \tau_{\text {ave }}\right) \times 10 \mathrm{fm}$, i.e., $\left(R \tau_{\text {ave }}\right)$ individual receptor-ligand binding events overlap each other.

Figures 4(b) and 4(c) plot the dependence of the white frequency parameter $h_{0}^{\prime}$ on $\tau_{\text {ave }}$ and $R$, respectively, which is derived from the numerical simulation. It is seen that $\log _{10} h_{0}^{\prime}$ goes up almost linearly as $\log _{10} \tau_{\text {ave }}$ and $\log _{10} R$ are increased. We suggest the following empirical formula

$$
h_{0}^{\prime}=I \delta t
$$

with the intensity of the spike-induced fluctuations

$$
I=\left(\Delta_{\text {ave }} / \omega_{0}\right)^{2},
$$

and the fluctuation time scale

$$
\delta t=\left[\frac{2}{1+\left(R \tau_{\mathrm{ave}}\right)^{-2}}\right]^{m} \sqrt{\frac{\tau_{\mathrm{ave}}}{R}},
$$

where the curve fitting gives $m=2 / 3$. By contrast, varying $\tau_{\text {ave }}$ hardly changes the random walk parameter $\log _{10} h_{-2}^{\prime}$ while $\log _{10} h_{-2}^{\prime}$ vs $\log _{10} R$ exhibits a linear behavior. One may write the following empirical formula

$$
h_{-2}^{\prime}=A I_{0} R \text {, }
$$

where $I_{0}=\left(10 \mathrm{fm} / \omega_{0}\right)^{2}$ and the curve fitting gives $A \approx 10$. We have also evaluated the cross times $\tau_{c 1}$ and $\tau_{c 2}$ from the numerical simulation [Fig. 4(b)]. It is found that $\tau_{c 1}$ rarely depends on $\tau_{\mathrm{ave}}$, corresponding to the behavior of $h_{-2}^{\prime}$. By 

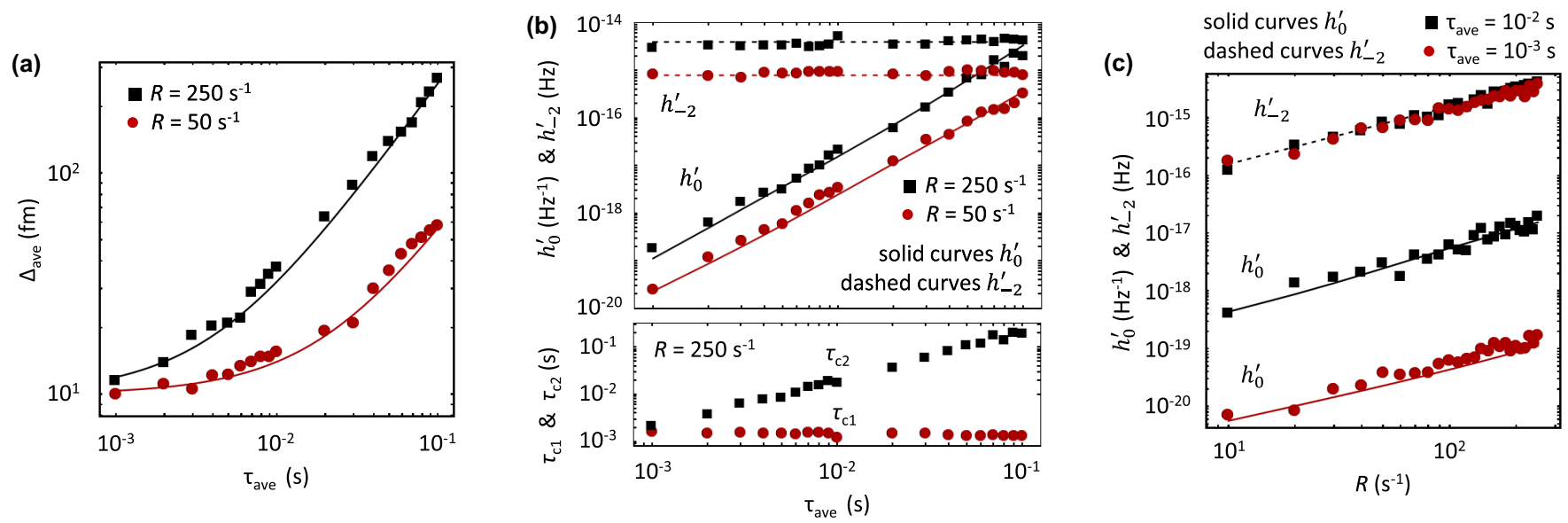

FIG. 4. (a) Dependence of the average spike height $\Delta_{\text {ave }}$ in a time trace of $\delta_{s}(t)$ on the average duration $\tau_{\text {ave }}$ with different event rates $R$. The solid lines correspond to the results of the empirical formula (18). (b) Upper panel: White frequency $h_{0}^{\prime}$ and random walk $h_{-2}^{\prime}$ parameters vs $R$. The solid lines correspond to the results of the empirical formula (19) while the dashed lines denote the results of the empirical equation (22). Lower panel: Cross times $\tau_{c 1}$ and $\tau_{c 2}$ as a function of $\tau_{\text {ave }}$ with $R=250 \mathrm{~s}^{-1}$. (c) White frequency $h_{0}^{\prime}$ and random walk $h_{-2}^{\prime}$ parameters vs $\tau_{\text {ave }}$. In all plots, the filled square and circle symbols denote the numerical simulation results.

contrast, $\tau_{c 1}$ scales as $\sqrt{h_{0}^{\prime}}$ since $\tau_{c 1}$ is determined by the cross between $\sqrt{h_{0}^{\prime} / 2 \tau}$ and $\sqrt{2 \pi^{2} h_{-2}^{\prime} \tau / 3}$.

Finally, we come to the question of how to derive the single-molecule event rate $R$ and the average duration $\tau_{\text {ave }}$ from the stability measurement of the mode frequency $\omega_{\mathrm{ccw}}(t)$. In experiment, it is challenging to extract the mode shift $\delta_{s}(t)$ from the time trace of $\omega_{\mathrm{ccw}}(t)$. Nevertheless, the values of $h_{0}, h_{0}^{\prime}, \tau_{c 1}$ and $\tau_{c 2}$ can be obtained directly from the Allan deviation of $\omega_{\mathrm{ccw}}(t)$ in the absence and presence of the receptor-ligand binding interactions. The random walk noise in $\delta_{s}(t)$ is then evaluated by $h_{-2}^{\prime}=h_{0}^{\prime} f_{c 2}^{2}$, where the cross frequency $f_{c 2}$ is given by Eq. (17). Using the empirical formulas (18)-(22), one obtains $R$ and $\tau_{\text {ave }}$ and further derive the kinetic rate constants $k_{\text {on }}$ and $k_{\text {off. It }}$ should be noted that the intrinsic fluctuations in $\omega_{\text {cav }}(t)$ need to be suppressed sufficiently so that $h_{0}$ and $h_{-2}$ are lower than $h_{0}^{\prime}$ and $h_{-2}^{\prime}$, respectively. Otherwise, the spike events are deeply submerged in the background noise. This requires a high $Q$ factor of the microsphere in the aqueous environment and a large polarizability of the ligand molecules.

\section{DISCUSSION}

In summary, we have numerically studied a frequencylocking-based single-molecule sensing scheme. The kinetic properties of a specific chemical/biological reaction may be assessed by simply measuring the Allan deviation of the frequency-locked probe beam. This approach is potentially applicable to an aqueous solution with a high ligand concentration and rapid receptor-ligand interactions. The current microcavity technology is ready to implement this sensing scheme.

Conventional optoplasmonic sensing is mainly based on the resonance frequency shift that is derived from the transmission spectrum of the WGM microcavity. To capture the complete WGM spectrum, the probe laser is slowly scanned over a wide spectral range around the WGM resonance frequency, resulting in a typical time resolution of $\sim 20 \mathrm{~ms}$. Such a relatively long time resolution of the detection restricts the relevant sensing applications. For instance, the conventional optoplasmonic sensors cannot respond to the rapid binding interactions between partially mismatched single DNA strands [9]. Also, the ligand concentration in an aqueous solution should be appropriately low (e.g., $\mathrm{fM} \sim \mathrm{nM}$ in [11-14]) so that the sensors can distinguish different single-molecule events. In comparison, the time resolution of the frequencylocking-based sensing scheme studied in this work is mainly determined by the feedback loop. The frequency locking time can be as short as $10 \mu \mathrm{s}$ [15], potentially enabling the sensors to monitor the rapid hybridization reactions of single stranded DNA molecules and identifying the single-molecule events with a high ligand concentration.

Besides the resonance-frequency-shift-based sensing mechanism, single molecules may also be detected through the mode splitting that results from the scatterer-induced coupling between $\mathrm{cw}$ and $\mathrm{ccw}$ WGMs [17]. The modesplitting mechanism enables the self-referenced detection, where the beat note between two split modes is measured, leading to a superior noise suppression [28]. Equation (3) is also applicable to study the mode-splitting mechanism. However, observing the mode splitting requires a high enough $Q$ (usually, $Q>10^{8}$ ) factor of the microsphere, which is challenging in the aqueous environment [5]. In addition, the polarizability $\alpha$ of the ligand molecules should be large enough to give rise to a strong indirect coupling between $\mathrm{cw}$ and $\mathrm{ccw}$ WGMs. This is quantified by the condition $\alpha>V_{\text {cav }} / Q$ [8]. For our specific sensor, the mode splitting resolvability demands $\alpha>7.2 \times 10^{-21} \mathrm{~m}^{3}$. Consequently, the mode-splitting mechanism significantly restricts its applications for detecting small-polarizability single molecules.

In our sensing scheme, the detection time is shortened by locking the probe beam to the microcavity, instead of directly monitoring the transmission spectrum of the microcavity as in conventional sensing schemes. Further shortening the detection time requires a faster capture of the complete WGM spectrum. This may be implemented by measuring the 
ring-up signal from the WGM microcavity that is driven by a sequence of far-detuned light pulses [29]. Cavity ring-up spectroscopy allows one to acquire single-molecule-induced effects at nanosecond timescales. Combining the Allan deviation analysis with the cavity ring-up technique potentially paves the path for the study of ultrafast biochemical reactions.

\section{ACKNOWLEDGMENTS}

We thank Narima Eerqing for sharing the experimental results and Gillian Fearnyough for reading through the paper. This work is supported by Engineering and Physical Sciences Research Council (EPSRC) Grant No. EP/R031428/1 (UK).

\section{APPENDIX: BACKSCATTERING AND RAYLEIGH SCATTERING}

In this section, we derive the equations of motion for the cw and ccw WGMs that are indirectly coupled through the backscattering and Rayleigh scattering of a tiny dipole scatterer (e.g., atom, molecule, and nanoparticle) located in the vicinity of the microcavity [17]. The scatterer is modeled by a two-state (upper $|e\rangle$ and lower $|g\rangle$ with an energy separation $\left.\omega_{A}\right)$ system with the relevant operators $\sigma_{e g}=|e\rangle\langle g|, \sigma_{g e}=$ $|g\rangle\langle e|$ and $\sigma_{z}=|e\rangle\langle e|-| g\rangle\langle g|$. The annihilation and creation operators for the cw (ccw) WGM are $a_{\mathrm{cw}}\left(a_{\mathrm{ccw}}\right)$ and $a_{\mathrm{cw}}^{\dagger}\left(a_{\mathrm{ccw}}^{\dagger}\right)$, respectively. The coupling strengths between the scatterer and two WGMs are

$$
g_{\mathrm{cw}, \mathrm{ccw}}=-\mathbf{d} \cdot \mathbf{u}_{\mathrm{cw}, \mathrm{ccw}}\left(\mathbf{r}_{0}\right) / \hbar,
$$

where $\mathbf{u}_{\mathrm{cw}, \mathrm{ccw}}(\mathbf{r})$ are the normalized mode vectors of two WGMs, $\mathbf{d}$ is the scatterer's dipole moment vector and $\mathbf{r}_{0}$ denotes the scatterer's location. The approximate solutions of $\mathbf{u}_{\mathrm{cw}, \mathrm{ccw}}(\mathbf{r})$ may be obtained from Ref. [30]. The scatterer also interacts with the (vacuum) bosonic reservoir, where the $j$ th boson mode is characterized by the oscillation frequency $\omega_{j}$, the annihilation $b_{j}$ and creation $b_{j}^{\dagger}$ operators and the boson mode-scatterer coupling strength

$$
g_{j}=\sqrt{\frac{\omega_{j}}{2 \hbar \varepsilon_{0} V_{\mathrm{vac}}}}|\mathbf{d}|,
$$

with the quantization volume $V_{\mathrm{vac}}$.

The Hamiltonian $H$ for the interacting system is written as

$$
\begin{aligned}
H / \hbar= & \omega_{\mathrm{cav}} a_{\mathrm{cw}}^{\dagger} a_{\mathrm{cw}}+\omega_{\mathrm{cav}} a_{\mathrm{ccw}}^{\dagger} a_{\mathrm{ccw}} \\
& +\frac{\omega_{A}}{2} \sigma_{z}+\sum_{j} \omega_{j} b_{j}^{\dagger} b_{j}
\end{aligned}
$$

$$
\begin{aligned}
& -\left[\sigma_{e g}\left(g_{\mathrm{cw}} a_{\mathrm{cw}}+g_{\mathrm{ccw}} a_{\mathrm{ccw}}\right)+\text { H.c. }\right] \\
& +\left[\sigma_{e g} \sum_{j} g_{j} b_{j}+\text { H.c. }\right] .
\end{aligned}
$$

In the Heisenberg picture, one may derive the following equations

$$
\begin{aligned}
\dot{a}_{\mathrm{cw}}= & \left(-\kappa_{0} / 2-i \omega_{\mathrm{cav}}\right) a_{\mathrm{cw}}+i g_{\mathrm{cw}}^{*} \sigma_{g e}, \\
\dot{a}_{\mathrm{ccw}}= & \left(-\kappa_{0} / 2-i \omega_{\mathrm{cav}}\right) a_{\mathrm{ccw}}+i g_{\mathrm{ccw}}^{*} \sigma_{g e}, \\
\dot{\sigma}_{g e}= & \left(-\gamma / 2-i \omega_{A}\right) \sigma_{g e}-i g_{\mathrm{cw}} a_{\mathrm{cw}} \sigma_{z} \\
& -i g_{\mathrm{ccw}} a_{\mathrm{ccw}} \sigma_{z}-i \sigma_{z} \sum_{j} g_{j} b_{j}, \\
\dot{b}_{j}= & -i \omega_{j} b_{j}+i g_{j}^{*} \sigma_{g e},
\end{aligned}
$$

where we have also inserted the cavity-loss and polarizationdecay (rate $\gamma$ ) terms. The above equations indicate that the $\mathrm{cw}$ and ccw waves are indirectly coupled through the photon scattering of the scatterer. In the weak-coupling limit, the scatterer is mainly in the lower state $|g\rangle$ and one has the approximation $\sigma_{z} \approx-1$. Further, we use the Weisskopf-Wigner approach to eliminate $\sigma_{g e}$ and $b_{j}[16]$ and arrive at

$$
\begin{aligned}
\left(\begin{array}{c}
\dot{a}_{\mathrm{cw}} \\
\dot{a}_{\mathrm{ccw}}
\end{array}\right)= & {\left[-\frac{\kappa}{2}-i \omega_{\mathrm{cav}}-\left(\frac{\Gamma}{2}-i \Delta\right)\right.} \\
& \left.\times\left(\begin{array}{cc}
1 & e^{i \phi} \\
e^{-i \phi} & 1
\end{array}\right)\right]\left(\begin{array}{c}
a_{\mathrm{cw}} \\
a_{\mathrm{ccw}}
\end{array}\right) .
\end{aligned}
$$

Here, $\phi$ is the phase difference between cw and ccw waves at $\mathbf{r}_{0}$ and the scatterer's polarizability is defined as

$$
\alpha=\frac{|\mathbf{d}|^{2}}{\hbar \varepsilon_{0}} \frac{1}{\omega_{A}-\omega_{\mathrm{cav}}-i \gamma / 2} .
$$

The Rayleigh scattering rate $\Gamma$ and frequency shift $\Delta$ caused by the scatterer are respectively given by

$$
\Gamma=\frac{\alpha^{2} \omega_{\mathrm{cav}}^{4}}{6 \pi(c / n)^{3} V_{\mathrm{cav}}}, \quad \Delta=\frac{\alpha \omega_{\mathrm{cav}}}{2 V_{\mathrm{cav}}},
$$

with the refractive index $n$ of the environment medium. Thus, the backscattering and Rayleigh scattering of the scatterer lead to the spectral shift and broadening of WGMs. We further replace the photon operators $a_{\mathrm{cw}, \mathrm{cc} w}$ with their $c$-number counterparts $\mathcal{A}_{\mathrm{cw}, \mathrm{ccw}}$ due to the classical laser lights in experiment. After extending the above analysis to a system containing multiple scatterers and including the prism-microcavity coupling, one obtains Eq. (3) in the main text.
[1] F. Vollmer and D. Yu, Optical Whispering Gallery Modes for Biosensing: From Physical Principles to Applications (Springer International Publishing, Cham, 2020).

[2] F. Vollmer, D. Braun, A. Libchaber, M. Khoshsima, I. Teraoka, and S. Arnold, Protein detection by optical shift of a resonant microcavity, Appl. Phys. Lett. 80, 4057 (2002).

[3] S. Arnold, M. Khoshsima, I. Teraoka, S. Holler, and F. Vollmer, Shift of whispering-gallery modes in microspheres by protein adsorption, Opt. Lett. 28, 272 (2003).
[4] F. Vollmer, S. Arnold, and D. Keng, Single virus detection from the reactive shift of a whispering-gallery mode, Proc. Natl. Acad. Sci. USA 105, 20701 (2008).

[5] M. L. Gorodetsky, A. A. Savchenkov, and V. S. Ilchenko, Ultimate $Q$ of optical microsphere resonators, Opt. Lett. 21, 453 (1996).

[6] D. K. Armani, T. J. Kippenberg, S. M. Spillane, and K. J. Vahala, Ultra-high- $Q$ toroid microcavity on a chip, Nature $\mathbf{4 2 1}$, 925 (2003). 
[7] M. Pöllinger, D. O'Shea, F. Warken, and A. Rauschenbeutel, Ultrahigh- $Q$ Tunable Whispering-Gallery-Mode Microresonator, Phys. Rev. Lett. 103, 053901 (2009).

[8] W. Kim, Ş. K. Özdemir, J. Zhu, and L. Yang, Observation and characterization of modesplitting in microsphere resonators inaquatic environment, Appl. Phys. Lett. 98, 141106 (2011).

[9] M. D. Baaske, M. R. Foreman, and F. Vollmer, Single-molecule nucleic acid interactions monitored on a label-free microcavity biosensor platform, Nat. Nanotech. 9, 933 (2014).

[10] J. D. Swaim, J. Knittel, and W. P. Bowen, Detection limits in whispering gallerybiosensors with plasmonic enhancement, Appl. Phys. Lett. 99, 243109 (2011).

[11] M. D. Baaske and F. Vollmer, Optical observation of single atomic ions interacting with plasmonic nanorods in aqueous solution, Nat. Photon. 10, 733 (2016).

[12] E. Kim, M. D. Baaske, and F. Vollmer, In situ observation of single-molecule surface reactions from low to high affinities, Adv. Mater. 28, 9941 (2016).

[13] E. Kim, M. D. Baaske, I. Schuldes, P. S. Wilsch, and F. Vollmer, Label-free optical detection of single enzyme-reactant reactions and associated conformational changes, Sci. Adv. 3, e1603044 (2017).

[14] S. Vincent, S. Subramanian, and F. Vollmer, Optoplasmonic characterisation of reversible disulfide interactions at single thiol sites in the attomolar regime, Nat. Commun. 11, 2043 (2020).

[15] G. Bianchini, P. Cancio, F. Minardi, F. S. Pavone, F. Perrone, M. Prevedelli, and M. Inguscio, Wide-bandwidth frequency locking of a 1083-nm extended-cavity DBR diode laser to a high-finesse Fabry-Pérot resonator, Appl. Phys. B 66, 407 (1998).

[16] A. Mazzei, S. Götzinger, L. de S. Menezes, G. Zumofen, O. Benson, and V. Sandoghdar, Controlled Coupling of Counterpropagating Whispering-Gallery Modes by a Single Rayleigh Scatterer: A Classical Problem in a Quantum Optical Light, Phys. Rev. Lett. 99, 173603 (2007).

[17] J. Zhu, S. K. Ozdemir, Y.-F. Xiao, L. Li, L. He, D.-R. Chen, and L. Yang, On-chip single nanoparticle detection and sizing by mode splitting in an ultrahigh- $Q$ microresonator, Nat. Photon. 4, 46 (2010).

[18] W. Chen, Ş. K. Özdemir, G. Zhao, J. Wiersig, and L. Yang, Exceptional points enhance sensing in an optical microcavity, Nature 548, 192 (2017).

[19] S. Subramanian, S. Vincent, and F. Vollmer, Effective linewidth shifts in single-molecule detection using optical whispering gallery modes, Appl. Phys. Lett. 117, 151106 (2020).

[20] D. W. Allan, Statistics of atomic frequency standards, Proc. IEEE 54, 221 (1996).

[21] F. Riehle, Frequency Standards: Basics and Applications (Wiley-VCH, Weinheim, 2004).

[22] A. Schliesser, G. Anetsberger, R. Rivière, O. Arcizetand, and T. J. Kippenberg, High-sensitivity monitoring of micromechanical vibration using optical whispering gallery moderesonators, New J. Phys. 10, 095015 (2008).

[23] M. Aspelmeyer, T. J. Kippenberg, and F. Marquardt, Cavity optomechanics, Rev. Mod. Phys. 86, 1391 (2014).

[24] W. J. Riley, Handbook of Frequency Stability Analysis, NIST Special Publication 1065 (NIST, Boulder, 2008).

[25] M. L. Gorodetsky and I. S. Grudinin, Fundamental thermal fluctuations in microspheres, J. Opt. Soc. Am. B 21, 697 (2004).

[26] G. Huang, E. Lucas, J. Liu, A. S. Raja, G. Lihachev, M. L. Gorodetsky, N. J. Engelsen, and T. J. Kippenberg, Thermorefractive noise in silicon-nitride microresonators, Phys. Rev. A 99, 061801(R) (2019).

[27] D. Yu and R. Dumke, Open Ising model perturbed by classical colored noise, Phys. Rev. A 100, 022124 (2019).

[28] B.-B. Li, W. R. Clements, X.-C. Yu, K. Shi, Q. Gong, and Y.-F. Xiao, Single nanoparticle detection using split-mode microcavity Raman lasers, Proc. Natl. Acad. Sci. USA 111, 14657 (2014).

[29] S. Rosenblum, Y. Lovsky, L. Arazi, F. Vollmer, and B. Dayan, Cavity ring-up spectroscopy for ultrafast sensing with optical microresonators, Nat. Commun. 6, 6788 (2015).

[30] B. E. Little, J.-P. Laine, and H. A. Haus, Analytic theory of coupling from tapered fibers and half-blocks into microsphere resonators, J. Lightwave Technol. 17, 704 (1999). 